\title{
New Robust Sensing Methods for DVB-T Signals
}

\author{
Chunyi SONG, M. Azizur RAHMAN and Hiroshi HARADA \\ New Generation Wireless Communications Research Center \\ National Institute of Information and Communications Technology (NICT) \\ 3-4 Hikarino-oka, Yokosuka, Kanagawa, 239-0847 Japan \\ Email: \{songe, aziz, harada\}@nict.go.jp
}

\begin{abstract}
The regulations for operation in the TV white space (TVWS) have been developed and released in USA and UK. It is believed that the ongoing TVWS related standardization projects, such as IEEE P802.19.1, IEEE P802.11af and IEEE P802.22, will encourage the regulation development in more countries and regions. Current regulations commonly require the $\mathrm{TV}$ band device (TVBD) or the sensing only TVBD to be capable of detecting incumbent TV signals of very low power level within a short time. To fulfill the strict requirements on both of sensitivity and sensing time while keeping the hardware implementation cost below a desired level, we propose two new sensing methods for DTV signals of DVB-T standard: the optimal one requires time synchronization and is therefore called The Proposed_Syn while the sub-optimal one requires no time synchronization and is called The Proposed_Asyn. Both computer simulation results and hardware testing results are shown in this paper. Simulation results show that for achieving a goal of high detection probability $(\geq 90 \%)$ and low false alarm probability $(\leq 1 \%)$ at a very low SNR $(=20 \mathrm{~dB})$ in AWGN channel, in comparison with conventional sensing methods, The Proposed_Syn reduces sensing time by $\mathbf{5 0 \%}$ and both proposed methods can significantly reduce hardware implementation cost by potentially reducing multiplexer number more than $99 \%$. Hardware sensing prototype testing results have further verified the improved performance by using the proposed sensing methods.
\end{abstract}

Keywords-Spectrum sensing; TV White Space; DVB-T; cognitive radio.

\section{INTRODUCTION}

Since The Federal Communications Commission (FCC) of United States (USA) approved the unlicensed use of TV white space (TVWS) in November 2008, we have seen many TVWS related activities from the other regulatory bodies, such as OFCOM of UK, CEPT in EU, MIC of Japan and iDA of Singapore [1]-[6]. Recently, The iDA has released a project to encourage TVWS technology trials in Singapore, which requires the TV band device (TVBD) to be capable of detecting TV signals of DVB-T standard and PAL standard at level of $120 \mathrm{dBm}$ in $8 \mathrm{MHz}$ channels [6]. In an $8 \mathrm{MHz}$ channel, the thermal noise power is around $-105 \mathrm{dBm}$; considering the hardware loss, noise level (NL) of a hardware sensing system may be around $-100 \mathrm{dBm}$, which means the SNR of received $120 \mathrm{dBm}$ TV signals is around $-20 \mathrm{~dB}$. Identifying the presence of a signal at a so low SNR level requires extremely robust sensing methods.

The most recent work showed that the cyclostationarity detector can achieve robust sensing till to $\mathrm{SNR}=-14 \mathrm{~dB}$ by utilizing multiple cyclic frequencies of OFDM signals used in many DTV signals, such as DVB-T and ISDB-T [7]. The sensing methods, which apply time domain sliding correlation and use the peak output for threshold decision, have shown better performance [8]-[9]. However, their sensing performance need to be further improved to fulfill above regulations' requirements on sensing, furthermore, the conducting of sidling correlation will lead to very high hardware implementation cost.

In this paper, we propose two new sensing methods for detecting DTV signals in low SNR regime: one applies sliding correlation over a sliding window that is selectively decided according to time synchronization results; the other one combines the signal feature detection and energy detection. The former achieves optimal performance and the latter achieves suboptimal performance. In comparison with the methods in [8]-[9], both proposed methods significantly reduce the hardware implementation cost; meanwhile, the optimal one significantly improves sensing performance and the sub-optimal one achieves similar performance with above conventional methods. Although the proposed sensing methods can also be applied to detect DTV signals of some other standards, we present them based on DVB-T signals for simplicity.

The rest of the paper is organized as follows. DVB-T signal specification is described in II. The proposed sensing algorithms are described in III. Simulation results and hardware test results are presented in IV. Finally the paper is concluded in V.

\section{DESCRIPTION OF DVB-T SIGNAL SPECIFICATION}

The physical layer characteristics of DVB-T signal can be referred to [10]. Here we only summarize main features of the signal concentrating on channel coding and modulation.

The DVB-T uses orthogonal frequency division multiplexing (OFDM) modulation and is organized in frames. One DVB-T signal is a super frame consists of 4 consecutive frames, with each frame consisting of 68 OFDM symbols. The continuous DVB-T signal in time domain is given by

$$
s(t)=e^{j 2 \pi f_{c} t} \sum_{p=0}^{3} \sum_{l=0}^{67} \sum_{k=0}^{852 m} C_{p, l, k} \psi_{p, l, k}(t)
$$

where the basic function $\varphi_{p, l, k}(t)$ is defined as 


$$
\psi_{p, l, k}(t)=e^{j 2 \pi(k-426 m) 896 m\left[t-T_{C P}-l T_{S}-68 p T_{S}\right] / 10^{6} \mathrm{~W}}
$$

whenever $(l+68 p) T_{s} \leq t \leq(l+68 p+1) T_{s}$ and 0 otherwise. Here, $T_{S}$ and $T_{C P}$ are, respectively, the duration of an OFDM symbol and the duration of cyclic prefix; $f_{c}$ is carrier frequency and $W$ is channel bandwidth in $\mathrm{MHz}$; the $m$ is signal mode index: it offers $2 \mathrm{~K}$ mode when $m=2$ and $8 \mathrm{~K}$ mode when $m=8$.

In DVB-T signals, some subcarriers, consists of around 10\% of total subcarriers, are transmitted at a higher power by about $2.5 \mathrm{~dB}$ than the other subcarriers. As shown in Fig.1, the subcarrier locations of boosted pilots repeat with the periodicity of four OFDM symbol durations.

\section{Proposed DVB-T SENSING METHODS}

\section{A. Sensing Performance Metrics}

In this work, we consider a single sensor based spectrum sensing. In a given frequency channel at a certain time in a certain area, there are two scenarios: either the primary signal is present or not. This is then formulated into following binary hypotheses:

$$
x(t)=\left\{\begin{array}{ll}
n(t) & \mathcal{H}_{0} \\
h(t) x_{0}(t)+n(t) & \mathcal{H}_{1}
\end{array} .\right.
$$

The sensing method also has two possible outcomes, as

$$
\begin{aligned}
& \mathcal{D}_{0} \text { : Primary signal not detected }, \\
& \mathcal{D}_{1} \text { : Primary signal detected. }
\end{aligned}
$$

The performance of a sensing method usually is evaluated in terms of probability of detection $\left(P_{D}\right)$, defined as the probability of detecting a primary signal whenever it is truly in the channel, i.e. $P_{D}=P\left(\mathcal{D}_{1} / \mathcal{H}_{1}\right)$, and probability of false alarm $\left(P_{F A}\right)$, defined as the probability of deciding the presence of a primary signal while in fact the channel is vacant, i.e. $P_{F A}=P\left(\mathcal{D}_{1} / \mathcal{H}_{0}\right)$.

\section{B. Synchronized Time Domain Correlation Detection}

The boosted pilot subcarriers repeart their subcarrier locations every four OFDM symbols. We then propose a reliable detection method by exploiting synchronized time domain correlation of the received DVB-T signals with a local reference signal that is the time domain expression of vector (3) over 4 OFDM symbol durations (4T).

\section{$>$ Correlation reference signal;}

We generate a reference sequence by conducting inverse Fourier transform (IFFT) of a vector that is only composed of the clean $(\mathrm{SNR}>30 \mathrm{~dB})$ boosted pilot sub-carriers, as

$$
s_{p}(t)=e^{j 2 \pi f_{c} t} \sum_{(l, k) \in A} C_{p, l, k} \psi_{p, l, k}(t),
$$

where the set $A$ defines positions of the boosted pilots. The $s_{p}(t)$ is deterministic as long as DVB-T parameters are known.

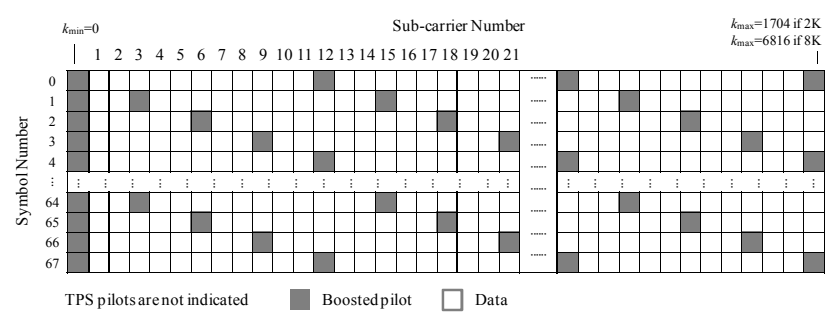

Fig.1 Locations of scattered boosted sub-carriers in DVB-T OFDM symbols ([10]). The horizontal axis represents the sub-carrier number (frequency) and the vertical axis represents the symbol number (time).

The boosted pilots repeat with the periodicity of $4 T$, we then sample $s_{p}(t)$ over $4 T$, as

$$
s_{p}[n]=s_{p}\left(n T_{s p}\right), n=0,1, \ldots N-1,
$$

where $1 / T_{\mathrm{sp}}$ is the sampling rate.

Time Synchronization;

The sliding correlation of received DVB-T sequence and the reference sequence in (5) over $4 T$ are given by

$$
R_{x p}(m)=\sum_{n=0}^{N-1} s_{p}(n) x^{*}(n+m), \quad m=0,1, \ldots, N-1 .
$$

To improve $R_{x p}(m)$, we can average received DVB-T sequence based on its periodicity of $N$, as

$$
x_{L}(n)=\frac{1}{L} \sum_{l=0}^{L-1} x(n-l * N), n=0,1, \ldots, N-1 .
$$

The Fig. 2 shows the normalized amplitudes of the $N$ outputs in (6), where SNR $>20 \mathrm{~dB}$ for the received DVB-T signals. Both methods in [8] and [9] achieve sensing decision based on the maximum output, $R_{x p}^{\max }=\max _{1 \leq \mathrm{m} \leq \mathrm{N}}\left(\left|R_{x p}(m)\right|\right)$. Such methods of using sliding correlation do nôt require time synchronization; however, they will lead to at least following two major weaknesses [11]. Firstly, the heavy real-time computation burden will result in high implementation cost and large hardware size, especially when the capability of simultaneously sensing multiple channels is required; secondly, in $\mathcal{H}_{0}$, the largest noise component is selected and which results in a large value of threshold for achieving a desired $P_{F A}$; in consequence it will degrade sensing performance. We then propose to use the synchronized time-domain correlation in spectrum sensing.

In (6), let $\mathcal{L}\left[R_{x p}^{\max }\right]$ denote the order of the output consisting of $R_{x p}^{\max }$ among $N$ outputs, and let the ratio of $\mathcal{L}\left[R_{x p}^{\max }\right] / N$ represent the position of $R_{x p}^{\max }$, then as shown in Fig.3, the oneto-one correspondence relationship exists between the position of $R_{x p}^{\max }$ and time offset. Therefore, by knowing the position of $R_{x p}^{\max }$ we can understand the time offset. By exploiting this feature, we develop the time synchronization method which 
realizes time offset estimation based on the distribution of position of $R_{x p}^{\max }$.

Using software, we conduct sliding correlation over $4 T$ and records $\mathcal{L}\left[R_{x p}^{\max }\right] / N$. By repeating the same operation for signals received at the interval of $\mathrm{K} \times 4 T(\mathrm{~K}=0,1,2, .$.$) , we can obtain the$ distribution of $\mathcal{L}\left[R_{x p}^{\max }\right] / N$. In the high SNR regime (e.g. $\mathrm{SNR}>0), \mathcal{L}\left[R_{x p}^{\max }\right] / N$ are consistent and then as the estimation results the time offsets are shown as one exact value. In our interested low SNR regime, owing to the impact of noise, in some cases $\mathcal{L}\left[R_{x p}^{\max }\right] / N$ may deviate from its true value and then their values are shown to distribute within a range. This deviation range changes at different values of SNR. In Fig.4, we use a metric called synchronization level to represent the deviation range. Assuming $\mathcal{L}\left[R_{x p}^{\max }\right] / N$ distributes between $M_{1} / N$ and $M_{2} / N$, where $M_{1}<M_{2}$, then the synchronization level is defined as

$$
\Gamma=1-\frac{M_{2}-M_{1}}{N} \leq 1 .
$$

The synchronization probability corresponding to synchronization level $\Gamma$ then is the probability of satisfying $M_{1} / N<$ $\mathcal{L}\left[R_{x p}^{\max }\right] / N<M_{2} / N$. As shown in Fig.4, in AWGN, $100 \%$ synchronization probability is achieved for aimed $\Gamma=1.0$ by averaging DVB-T signals over its periodicity more than three times $(L>3)$. In the one-path Rayleigh fading channel, synchronization probability improves around 0.065 when the aimed $\Gamma$ is loosed from 1.0 to 0.975 without conducting averaging operation $(L=1)$; this difference decreases when increasing $L$ and it becomes neglectable when $L>13$.

\section{$>$ Synchronized Time-domain Correlation;}

Based on assumption in (8), we conduct sliding correlation over the reduced window of $\left[M_{1}, M_{2}\right]$, as

$$
R_{x p}(m)=\sum_{n=0}^{N-1} s_{p}(n) x^{*}(n+m), m=M_{1}, M_{1}+1 \ldots, M_{2} .
$$

As shown in Fig.5,

1) when $\Gamma=1$, i.e. $M_{2}=M_{1}$, the perfect synchronizaton is achieved, then computation in (9) becomes a simple correlation and the only output is used for threshold decision.

2) When $M_{2}>M_{1}$, the maximum output among $M_{2}-M_{1}+1$ outputs is selected for threhold decision.

Since the sliding window is selectively decided according to the $\Gamma$, we call computation in (9) the selective sliding correlation.

In comparison with the sliding correlation based sensing methods developed in [8]-[9], The Proposed_Syn has decreased multiplexer number from $N^{2}$ to $\left(M_{2}-M_{1}+1\right) \times N$ for one hardware sensing system; in addition, The Proposed_Syn reduces the possibility of making decision based on the peak noise component in $\mathcal{H}_{0}$; in consequence, the sensing performance is also expected to be improved.

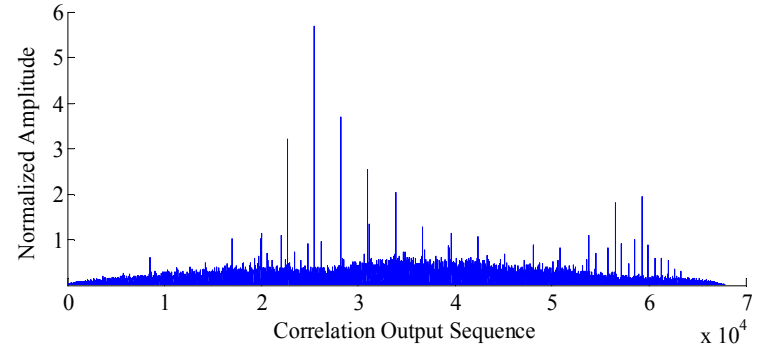

Fig.2 Normalized amplitudes of sliding correlation outputs.

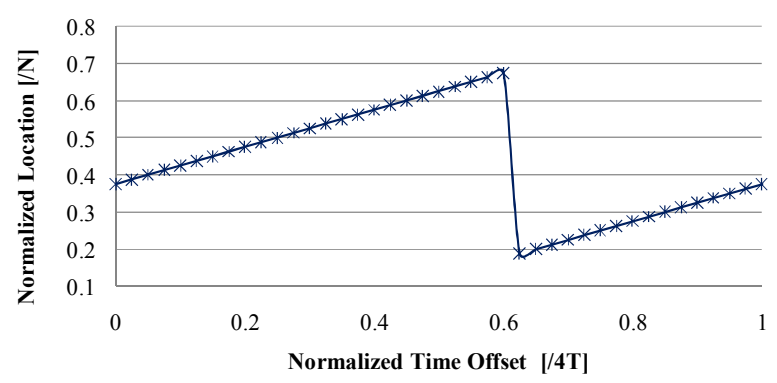

Fig.3 The $\mathcal{L}\left[R_{x p}^{\max }\right]$ vs. time offset.

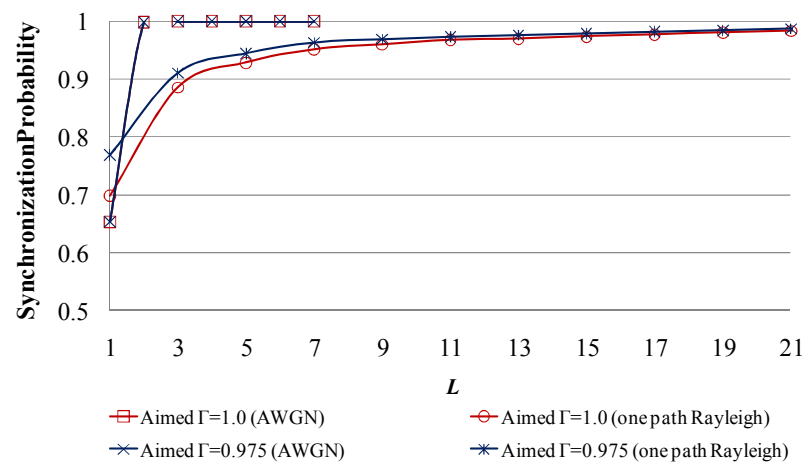

Fig.4 Synchronization probability vs. $L$ of (7), SNR=-20dB.

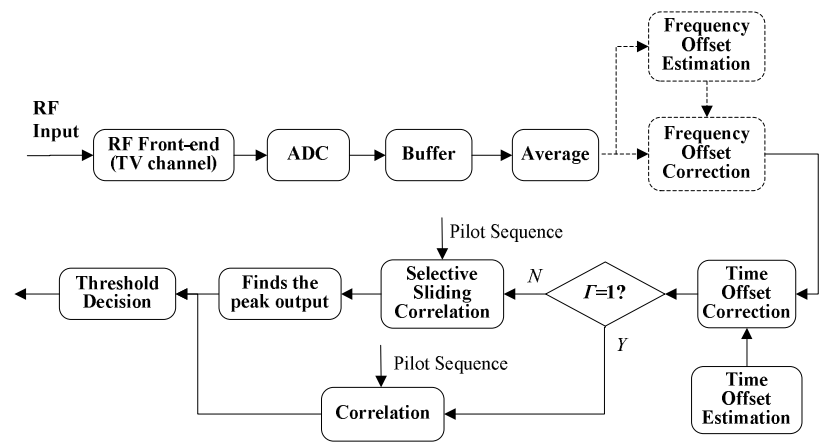

Fig.5 Simplified block diagram of The Proposed_Syn.

In The Proposed_Syn, the sliding correlation used in time synchronization stage is realized using software; in a channel with fast changing status (between $\mathcal{H}_{0}$ and $\mathcal{H}_{1}$ ), the software 
processing is too time consuming $(>>1 s)$. We then propose another sensing method of requiring no time synchronization as the solution for fast changing channel. To distinguish it from The Proposed_Syn, we call it The Proposed_Asyn.

\section{Description of The Proposed_Asyn}

Fig.6 shows the simplified block diagram of The Proposed_Asyn. Without knowing the timing, the received DVB-T sequences are firstly multiplied with the pilot sequence defined in (5) and then the absolute products are integrated, as:

$$
R_{x p}=\sum_{n=0}^{N-1}\left|s_{p}(n) x(n)\right| \quad .
$$

The Proposed_Asyn can be regarded as the combined signal feature detection and energy detection. To further improve the sensing reliability, we conduct computation of (10) for multiple times by delaying the signal reception time a random interval, $\tau_{i}$, for any subsequent operation, as:

$$
\bar{R}_{x p}=\frac{1}{\kappa} \sum_{J=1}^{\kappa}\left[\sum_{n=0}^{N-1}\left|s_{p}(n) x\left(n+\sum_{i=1}^{J} \tau_{i}\right)\right|\right]
$$

subjects to

$$
\sum_{i=1}^{K}\left(\tau_{i}\right)=4 T
$$

Therefore, the basic sensing time (sample duration) is $8 T$.

\section{PERformance Evaluation}

The performance of the proposed two sensing methods are evaluated by computer simulation [12], and then performance of a hardware sensing prototype implementing The Proposed_Asyn is also shown. The achieved sensing performance is compared with that of the conventional sensing method developed in [9].

\section{A. Computer Simulation Performance}

The Clean RF DVB-T signals (SNR $>30 \mathrm{~dB}$ ) generated by the signal generator (SG) are directly frequency down converted and sampled, the samples are further processed in Matlab to achieve low SNR. The main simulation parameters are as follows:

- In an $8 \mathrm{MHz}$ channel centered at $634 \mathrm{MHz}$

- Simulation runs for each SNR: 10,000

- $P\left\{H_{0}\right\}=P\left\{H_{1}\right\}=0.5$

- The Sensing Goal: $P_{D} \geq 0.9$ and $P_{F A}=0.01$ at $\mathrm{SNR}=-20 \mathrm{~dB}$.

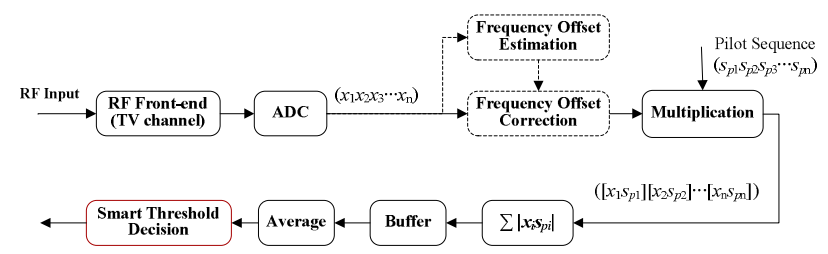

Fig.6 Simplified block diagram of The Proposed Asyn.
Figures 7-8 show the $P_{D}$ vs. SNR and the receiver operating characteristic (ROC) curves of The Proposed_Syn. The results verify that The Proposed Syn outperforms the conventional sensing method, and that the performance of The Proposed_Syn can be improved by improving $\Gamma$. To achieve The Sensing Goal, as shown in Fig.7, in AWGN, The Proposed_Syn at $\Gamma=0.975$ does not need to average received signals as defined in (7) $(L=1)$, while the conventional sensing method requires $L \geq 2$; as shown in Fig.8, in one-path Rayleigh fading channel, The Proposed_Syn requires $L \geq 5$ at $\Gamma=0.975$ and $L \geq 2$ at $\Gamma=1.0$, in comparison with $L \geq 7$ required by the conventional method.

The Fig.9 demonstrates the sensing performance of The Proposed_Asyn by setting $\boldsymbol{\kappa}$ defined in (11) to four different values. The results tell that the performance can be improved by increasing $\boldsymbol{\kappa}$ and that The Sensing Goal is achieved when $\boldsymbol{\kappa} \geq 16$.

\section{B. Performance of Hardware Sensing Prototype}

We implemented The Proposed Asyn into a hardware sensing system. As described in (11) and (12), the increase of $\boldsymbol{\kappa}$ does not necessarily lead to increasing the sensing time; in the sensing system shown in Fig.10 we set $\boldsymbol{\kappa}=64$.

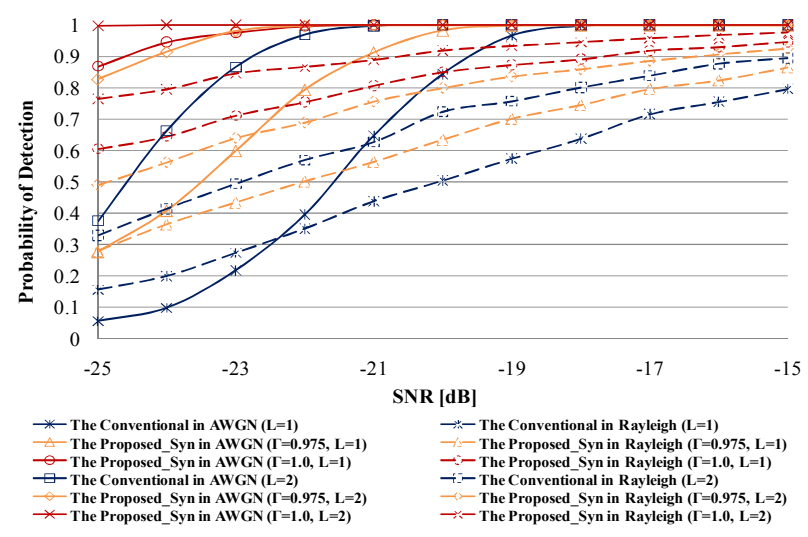

Fig.7 $\quad P_{D}$ vs. SNR: $P_{F A}=0.01$. $2 K$ mode DVB-T, $T_{C P}=T / 32$.

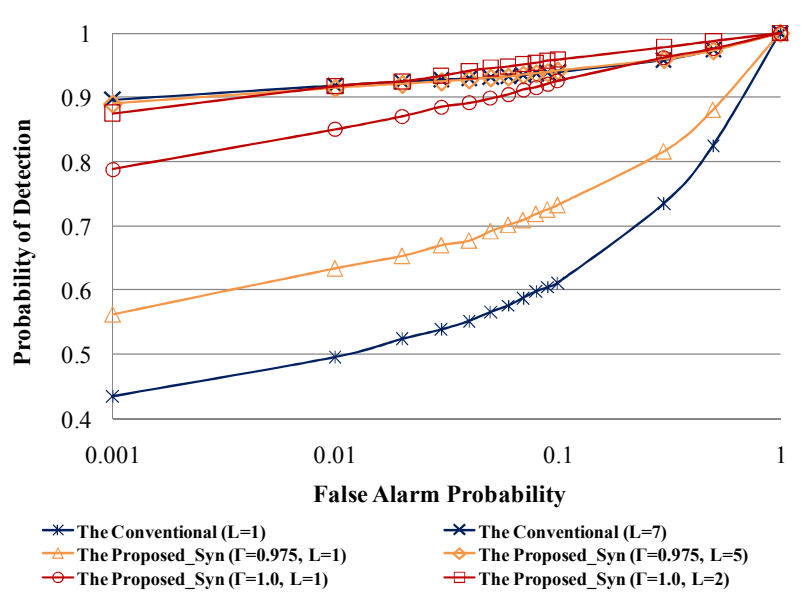

Fig.8 ROC curve: in one-path Rayleigh fading channel, $\mathrm{SNR}=-$ 20dB. $2 K$ mode DVB-T, $T_{C P}=T / 32$. 
In testing the sensitivity, we generate RF DVB-T signals using a signal generator and then input them into the sensing system through a cable. Over an $8 \mathrm{MHz}$ channel centered at 634 $\mathrm{MHz}$, we record the sensing results for three input levels by running 50,000 sensing runs for each input level. Since the system noise level of the sensing prototype is around $-100 \mathrm{dBm}$, the SNR of received signal is around $-20 \mathrm{~dB}$ [13].

The results in Fig.12 further verify that The Proposed_Asyn can achieve robust sensing of DVB-T in very low SNR regime.

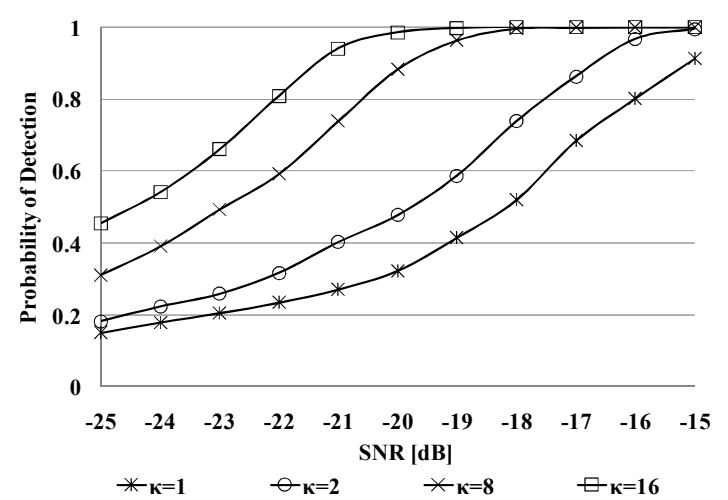

Fig.9 $\quad P_{D}$ vs. SNR: The Proposed_Asyn, $P_{F A}=0.1$. The $8 K$ mode DVB-T, $T_{\mathrm{CP}}=T / 8$.

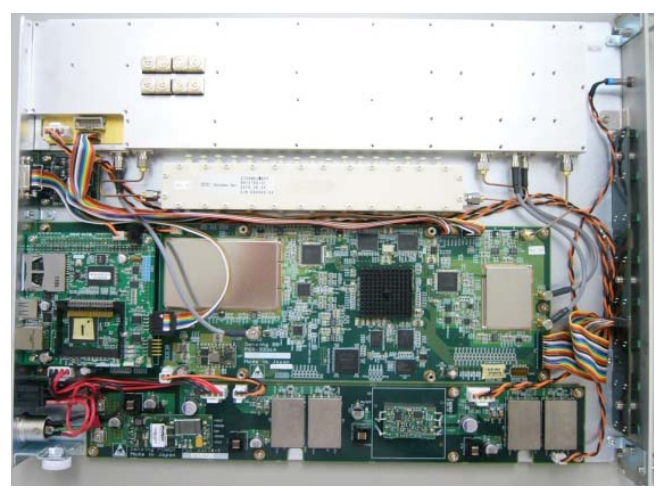

Fig.10 NICT Hardware Sensing System.

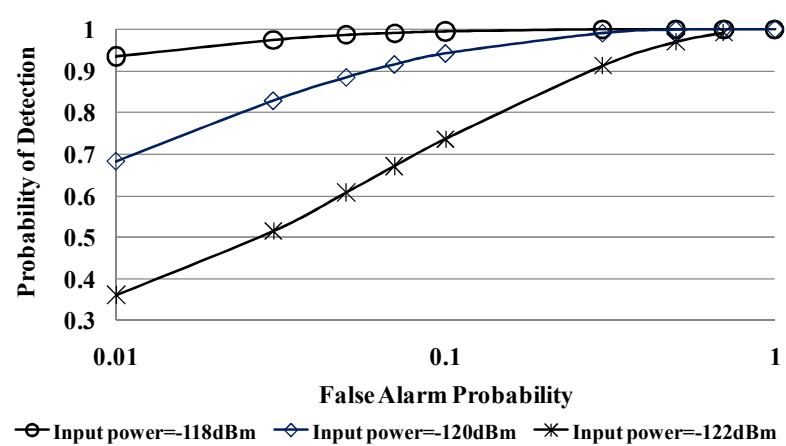

Fig.11 ROC curve: The Proposed_Asyn; the 8K mode DVB-T, $T_{\mathrm{CP}}=T / 8$

\section{CONCLUSION}

In this paper, we presented two new sensing methods for reliable sensing of DVB-T in very low SNR regime within short time. In computer simulations, the one achieves sensing using synchronized time-domain correlation has shown better sensing performance over the conventional sensing method, and reduces sensing time by $50 \%$ in AWGN channel. The one achieves sensing using the combined feature and energy detection has also shown the capability of reliable sensing DVB-T signals at $\mathrm{SNR}=-20 \mathrm{~dB}$. In addition, both proposed sensing methods reduce multiplexer by more than $99 \%$ in the hardware implementation in comparison with the conventional sensing method.

The second proposed sensing method has also been implemented into the hardware sensing system and the sensing test results are also shown in this paper. The results have further verified its robustness in very low SNR regime.

\section{ACKNOLEDGMENT}

This research was conducted under a contract of R\&D for radio resource enhancement, organized by the Ministry of Internal Affairs and Communications, Japan.

\section{REFERENCES}

[1] Federal Communication Commission Document 08-260: "Second report and order and memorandum opinion and order: In the Matter of Unlicensed Operation in the TV Broadcast Bands," Nov. 14, 2008.

[2] Ofcom, "Digital Dividend: Cognitive access statement on licence-exempting cognitive devices using linterleaved spectrum," July 2009. Available at: http://www.ofcom.org.uk/consult/condocs/cognitive/ tatement/statement.pdf.

[3] CEPT, "Terms of Reference SE43," Available at: http://www.cept. org/F113EB40-7941-455A-952B-EC04A54C8B2.W5Doc? frames= nol\&

[4] Cognitive Radio Systems for Efficient Sharing of TV White Spaces in European Context, available at: http://www.ict-cogeu.eu/.

[5] MIC, Japan, "Examination Team For New Radio usage Vision," Communication News, Vol.20, no.20, January 2010.

[6] iDA, "Trial of White Space Technology Accessing VHF and UHF Bands in Singapore (Information Pack)," available at: http://www.ida.gov.sg/doc/ Policies\%20and\%20Regulation/Policies_and_Regulation_Level2/WST/ WhiteSpaceRegFW.pdf.

[7] M. Kim, P. Kimtho and J. Takada, "Performance enhancement of cyclostationarity detector by utilizing multiple cyclic frequencies of OFDM signals," Proc. IEEE DySPAN 2010.

[8] Danyo Danev, "On Signal Detection Technique for the DVB-T Standard," Proc.ISCCSP 2010.

[9] Vasanth Gaddam and Monisha Ghosh, "Robust Sensing of DVB-T Signals," IEEE DySPAN 2010.

[10] ETSI EN 300744 V1.6 .1 (2009-01), "Digital video broadcasting (DVB); framing structure, channel coding and modulation for digital terrestri al television," ETSI, Tech. Rep., 2009.

[11] Chunyi SONG, et al, "Robust Spectrum Sensing of DVB-T Signals," IEICE Technical Report on Software Radio, pp:161-168, Oct. 2010.

[12] H. Harada and R. Prasad, Simulation and Software Radio For Mobile Communications. Artech House, 2002.

[13] Chunyi SONG, Azizur M.Rahman, Hiroshi HARADA, "Sensing methods for DVB-T,” IEEE 802.22 document: IEEE 802.22-11/0015r1, Feb. 2011. 